XIV Simpósio Nacional de Sistemas Prediais

Gestão, Eficiência e Sustentabilidade

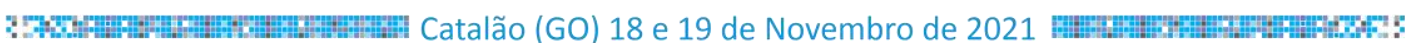

\title{
MÉTODO DE VERIFICAÇÃO EM CAMPO DE PRESSÕES DINÂMICAS EM PONTOS DE CONSUMO DE SISTEMAS PREDIAIS DE ÁGUA FRIA E QUENTE
}

\section{Method of field verification of dynamic pressure in points of use for cold and hot water supply}

\author{
YAMADA, Marco Antonio Furtado; OLIVEIRA, Lúcia Helena de ${ }^{2}$
}

Recebido em 06 de agosto de 2021, aprovado em 06 de setembro de 2021, publicado em 18 de novembro de 2021

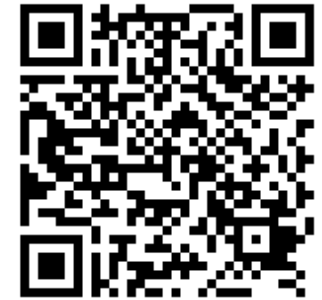

Palavras-chave: Comissionamento;

Sistemas prediais de água fria quente;

Uso eficiente da água.

Keywords:

Commissioning;

Hot water systems;

Water efficiency.
RESUMO: O comissionamento é uma inovação que requer alteração no processo de projeto. Além disso, devido a revisão e integração das normas técnicas NBR 5626 (ABNT, 1998) e NBR 7198 (ABNT, 1993) no ano de 2020, diversos requisitos foram atualizados e novos foram estabelecidos. $A$ soma desses fatores criou a necessidade de pesquisas devido à dificuldade em integrar o comissionamento no processo de projeto dos sistemas prediais. Dentre as dificuldades encontradas, a etapa de verificação em campo da conformidade dos requisitos e critérios de desempenho é um dos pontos que precisa ser abordado. Parte dessa dificuldade ocorre devido à falta de métodos de verificação claramente definidos. Desse modo, este artigo propõe um método de verificação dos critérios de desempenho da distribuição de água do sistema predial de água fria e quente. O método foi desenvolvido com base em uma pesquisa exploratória sobre os temas relacionados, além de uma etapa em campo, onde o método e a instrumentação para a verificação foram empregados. Os resultados indicam que o método é capaz de verificar os critérios estabelecidos, porém a instrumentação precisa ser melhorada para verificar outros critérios que podem ser estabelecidos pelo Proprietário do Projeto.

ABSTRACT: The commissioning process is an innovation that requires changes in the design process. Besides that, due to the revision and integration of the technical standards NBR 5626 (ABNT, 1998) and NBR 7198 (ABNT, 1993) in the year of 2020, many requirements were updated and new ones were set. The sum of those factors created the need of research due to the difficulty of integrating the commissioning process in the design process of building systems. Amongst the difficulties encountered, the assessment of performance requirements and criteria on the delivery and operation phases must be addressed. In addition, part of the difficulties occurs due to the lack of clear methods of performance assessment. Therefore, this paper proposes a method of verification of performance criteria for water distribution in cold and hot water building systems. The method was developed based on exploratory research of the related themes, as well as a field application of the method and measuring equipment. The results show that the method can verify the performance criteria stablished, however the measuring instruments require improvement to allow the verification of other criteria that can be required by the Project Owner.

\section{CONTATO DOS AUTORES:}

${ }^{1}$ YAMADA, Marco Antônio Furtado: Axiom Engenharia, marco.yamada@axiomengenharia.com.br

2 OLIVEIRA, Lúcia Helena de: Escola Politécnica da Universidade de São Paulo, lucia.helena@usp.br 


\section{INTRODUÇÃO}

O comissionamento dos sistemas prediais hidráulicos e sanitários ainda é uma inovação no processo de projeto, de modo que ele necessita de mais pesquisas aprofundadas sobre diversas atividades. Além disso, em função da atualização e integração das normas técnicas NBR 5626 (ABNT, 1998) e NBR 7198 (ABNT, 1993), que regiam, respectivamente, sistemas de água fria e quente, na nova NBR 5626 (ABNT, 2020), diversos aspectos que foram modificados também necessitam de pesquisas mais aprofundadas.

Dentre as diversas atividades que precisam ser executadas para a realização do comissionamento e presentes na NBR 5626 (ABNT, 2020), verifica-se que algumas delas não possuem requisitos, critérios e métodos de verificação claramente definidos, como, por exemplo, a capacidade do sistema de aquecimento em fornecer água quente e a verificação da pressão dinâmica exigida nos pontos de consumo entre outras.

Um dos aspectos fundamentais do desempenho dos sistemas prediais de água quente e fria é no âmbito da distribuição. Neste artigo, o termo "distribuição de água quente e fria" se refere a capacidade do sistema em fornecer água de modo adequado. O termo "adequado" implica que a água deve ser fornecida dentro das faixas de pressão, vazão, temperatura e intervalos compatíveis com as atividades e processos consumidores definidos, considerando as condições de uso simultâneo, tempo de espera pela água quente e as oscilações máximas especificadas no ponto de utilização (YAMADA e OLIVEIRA, 2020).

Nota-se que este requisito, fundamental ao desempenho do sistema, não possui critérios e métodos de verificação claros descritos nas normas técnicas, de modo a garantir que as especificações de projeto sejam passíveis de verificação durante a fase de entrega e após o início da operação do sistema.

Assim, no caso de uma suspeita de falha nesse requisito, ou mesmo para fins de verificação de conformidade, não existem métodos padronizados para a sua verificação. Desse modo, este artigo propõe um método para a medição de pressões dinâmicas em campo para contribuir com o desenvolvimento das atividades de comissionamento.

\section{OBJETIVO}

O presente artigo tem como objetivo propor um método de medição em campo das pressões dinâmicas de água em pontos de consumo, de modo a permitir a verificação da conformidade dos requisitos e critérios de desempenho relativos a distribuição de água durante o comissionamento dos sistemas prediais de água fria e quente.

\section{REFERENCIAL TEÓRICO}

\subsection{Critérios de desempenho para a verificação da distribuição de água em pontos de consumo}

Considerando-se que os critérios de desempenho necessitam ser definidos em projeto, propõe-se o estabelecimento dos critérios abaixo para a verificação do requisito:

- a pressão dinâmica mínima no ponto de consumo;

- a faixa de vazão de cada um dos pontos a serem verificados;

- a condição de simultaneidade prevista; 
- a temperatura da água quente e da água misturada.

Adicionalmente, recomenda-se estabelecer:

- o tempo máximo de espera;

- as oscilações máximas de pressão, temperatura da água misturada e das vazões nos pontos de consumo.

Para a análise de oscilações, os medidores de vazão, temperatura e pressão necessitam ser eletrônicos e devem permitir o ajuste do intervalo de captação e leitura dos dados obtidos. Considerando que as oscilações de pressão, vazão e temperatura podem ocorrer de modo brusco e ter uma duração reduzida, recomenda-se que o intervalo de captação dos dados pelos sensores não seja superior a 1 segundo.

\subsection{Métodos de ensaios de verificação de vazões e pressões de operação de equipamentos sanitários}

As diversas atividades relacionadas ao uso de água quente e fria são executadas pelos usuários, quer sejam para fins sanitários, quer por equipamentos específicos, a exemplo de máquinas de lavar roupas e louças. A capacidade do sistema em fornecer água para esses equipamentos está diretamente ligada à disponibilidade de pressão dinâmica e à vazão necessária para a realização de uma atividade.

Assim, para avaliar se um determinado equipamento terá o desempenho esperado, o método de verificação deve ser capaz de medir a vazão e as pressões dinâmicas nos pontos de consumo.

Devido às diferentes características dos equipamentos sanitários, o método de verificação, preferencialmente, deve ser capaz de ser executado sem a presença do equipamento sanitário, de modo a verificar as condições do sistema independentemente do aparelho que será instalado posteriormente.

Adicionalmente, os projetos de sistemas prediais hidráulicos e sanitários geralmente contemplam a disponibilidade de vazão e de pressão dinâmica no ponto de consumo, sem a instalação do aparelho sanitário, uma vez que o modelo e suas características muitas vezes não são conhecidos na etapa de projeto.

Para o desenvolvimento da instrumentação de teste realizou-se uma pesquisa exploratória nas normas dos aparelhos sanitários mais empregados nos sistemas prediais de água fria e quente, de maneira a verificar os métodos de ensaio normativos para a definição das pressões e vazões. Nas Figuras 1, 2 e 3 ilustra-se a instrumentação para a verificação desse requisito nas normas técnicas dos aparelhos. 
Figura 1 - Instrumentação prescrita pela NBR 16749 para verificação da vazão mínima de misturadores duplocomando

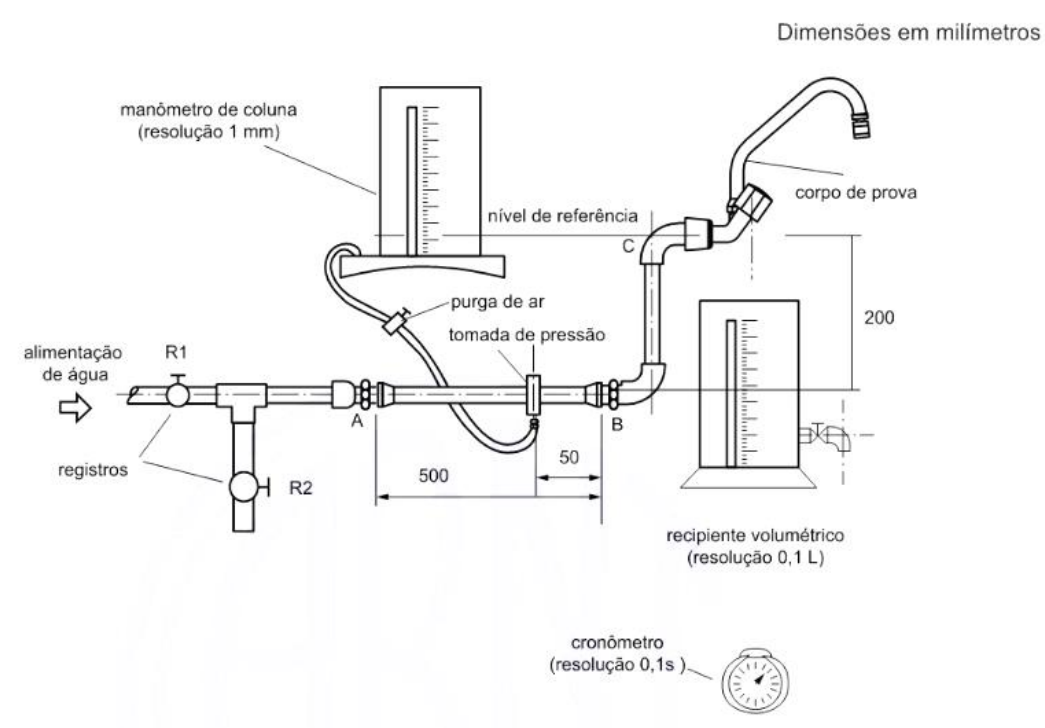

Fonte: NBR 16749 (ABNT, 2019).

Figura 2 - Instrumentação prescrita pela NBR 15267 para verificação da vazão mínima de misturadores monocomando

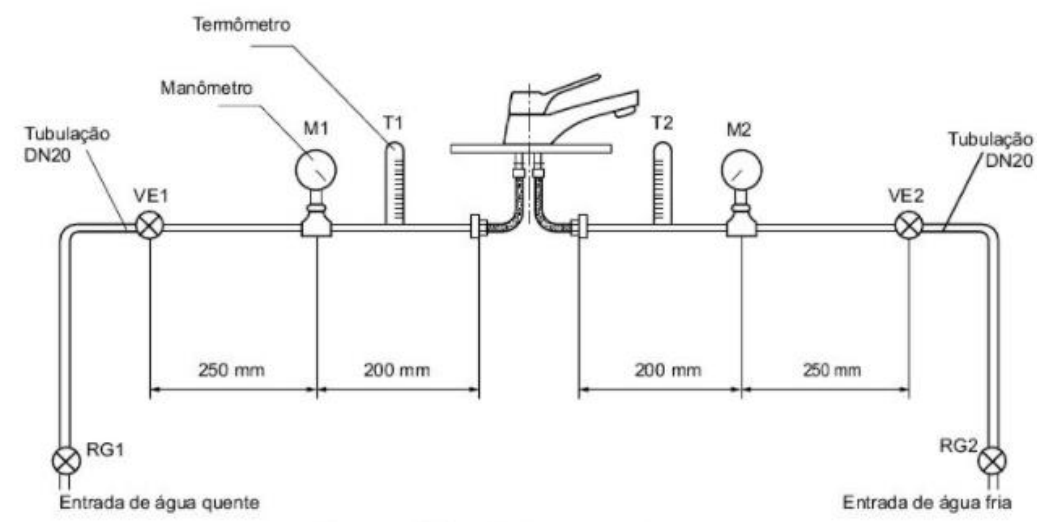

Fonte: NBR 15267 (ABNT, 2017).

Figura 3 - Instrumentação prescrita pela NBR 15206 para verificação da vazão mínima de chuveiros

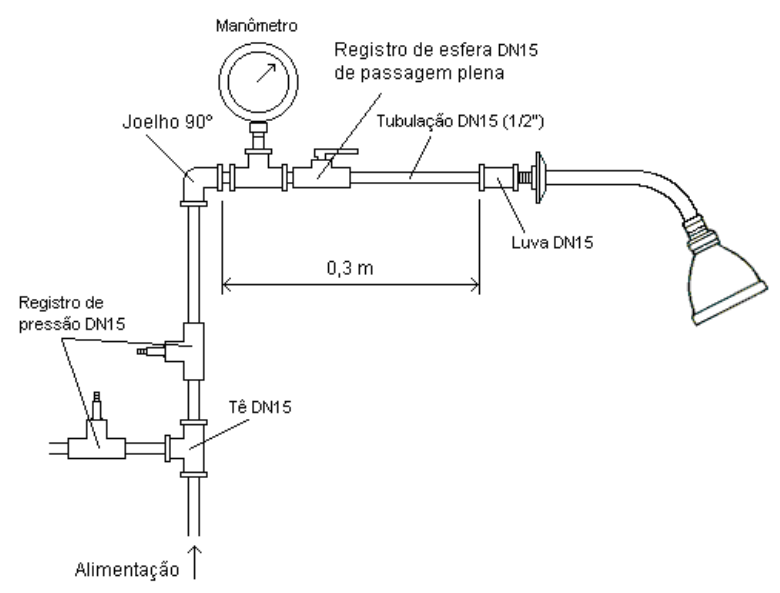

Fonte: NBR 15206 (ABNT, 2005). 
Nota-se que os ensaios normativos verificam apenas a vazão mínima e não foram desenvolvidas para a verificação dessa grandeza em campo. Considerando-se que o objetivo é verificar a vazão e a pressão dentro das condições de operação previstas ou existentes na edificação, é necessário modificar a instrumentação de teste.

\section{MATERIAIS E MÉTODOS}

\subsection{Materiais}

Após a verificação da aparelhagem empregada para a verificação das normas técnicas, um sistema de medição de vazão e pressão dinâmica foi desenvolvido para ser instalado em campo. O cronômetro e o recipiente volumétrico foram substituídos por um medidor de vazão, um armazenador de dados e um componente de interface, ilustrados na Figura 4(a), $4(b)$ e 4 (c), respectivamente.

\section{Figura 4 - Componentes do sistema de medição de vazão}

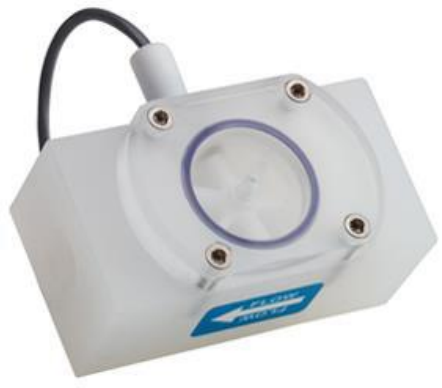

(a)

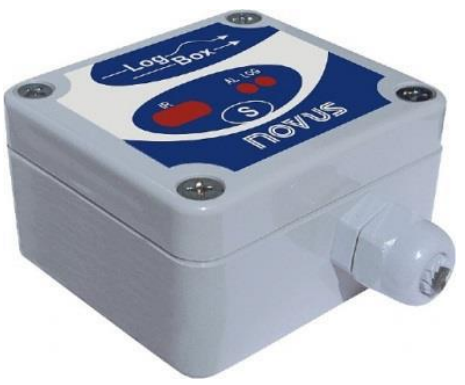

(b)

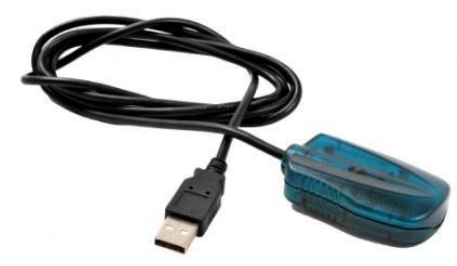

(c)

Fonte: Yamada (2021)

O sistema de medição de vazão foi instalado com um manômetro. A temperatura foi verificada com um termômetro, posicionado no jato de água, cerca de 30 a $40 \mathrm{~cm}$ abaixo do ponto de conexão do sistema de medição. Idealmente, os equipamentos de medição de temperatura e pressão deveriam ser eletrônicos e ligados no mesmo armazenador de dados, de modo que as leituras fossem simultâneas.

Considerando que, geralmente, os pontos críticos de consumo são os chuveiros, o sistema de medição, ilustrado na Figura 5, foi montado para fazer a avaliação em campo.

Figura 5 - Esquema do sistema de medição de vazão em chuveiros

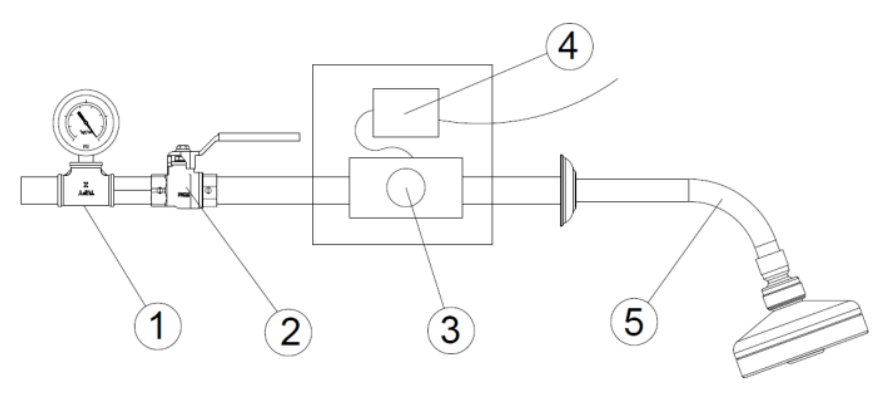

\begin{tabular}{|c|l|}
\hline & \multicolumn{1}{|c|}{ Componente } \\
\hline 1 & Manômetro \\
\hline 2 & Válvula de esfera \\
\hline 3 & Medidor de vazão \\
\hline 4 & Armazenador de dados (Datalogger) \\
\hline 5 & Chuveiro de vazão limitada (12 L/min) \\
\hline
\end{tabular}

Fonte: Yamada (2021)

\subsection{Procedimento de verificação}

A verificação foi feita por meio do seguinte procedimento: 
- abrir totalmente o registro de água quente e medir a temperatura da água;

- abrir gradualmente o registro de pressão de água fria de modo que a temperatura da água misturada alcance o valor estabelecido no critério;

- regular a vazão da água misturada por meio da válvula de esfera (2);

- repetir o procedimento para cada um dos pontos de consumo descritos na condição de simultaneidade, corrigindo as temperaturas e vazões antes de iniciar a leitura;

- calcular as vazões médias, desconsiderando o tempo de ajuste;

- anotar as pressões dinâmicas de cada ponto.

\subsection{Verificação em campo}

O sistema de medição foi instalado em um edifício residencial com 25 pavimentos, sendo que cada pavimento possui 4 unidades. Cada unidade possui dois banheiros, uma cozinha e uma área de serviço. Um dos banheiros é o ponto menos favorável do sistema devido a maior extensão linear dos ramais. O sistema de distribuição de água é composto por 4 zonas de pressão, conforme descrito no Quadro 1.

\section{Quadro 1 - Zonas de pressão}

\begin{tabular}{|c|c|}
\hline Pavimento & Zona \\
\hline $25-20$ & Pressurizada (P) \\
\hline $19-14$ & Gravitacional (G) \\
\hline $13-4$ & Reduzida 1 (R) \\
\hline $3-$ Térreo & Reduzida 2 (RR) \\
\hline
\end{tabular}

Fonte: Yamada (2021).

Após a entrega das unidades diversos moradores alegaram que a vazão dos chuveiros era insuficiente. Devido a isso, a construtora e a incorporadora decidiram investigar as ocorrências. Os testes foram realizados nas unidades com menor pressão estática e naquelas que apresentaram maior número de reclamações. A instalação do sistema de medição está ilustrada na Figura 6.

Figura 6 - Instrumentação instalada em campo

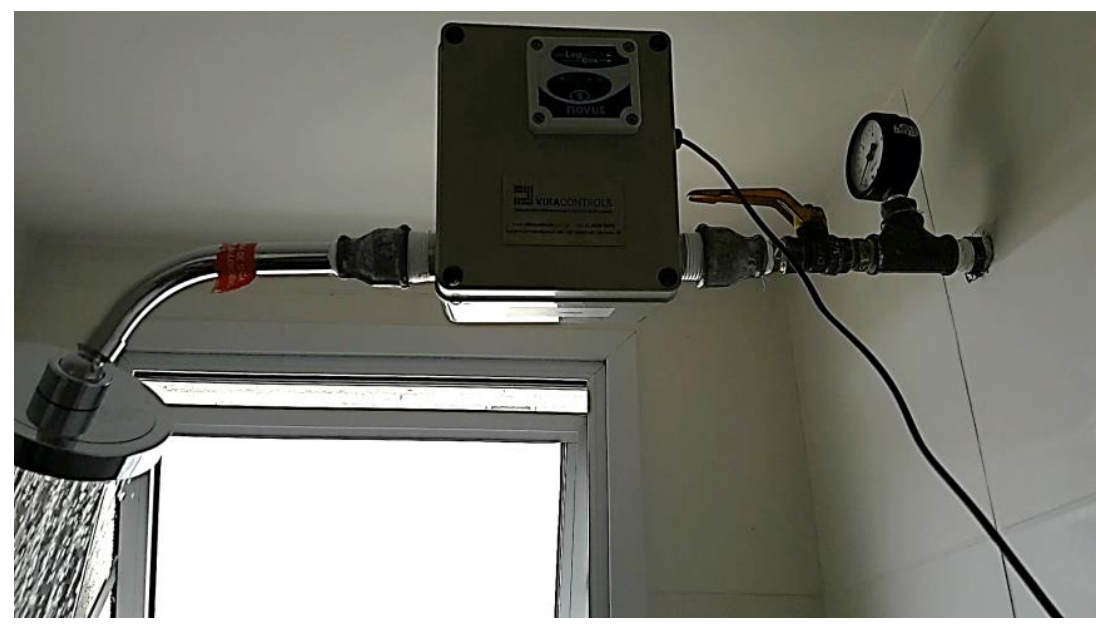

Fonte: Yamada (2021). 
Para melhor entendimento do comportamento do sistema foram instalados manômetros na entrada e na saída do aquecedor, bem como um termômetro na saída do aquecedor, conforme demonstrado na Figura 7.

Figura 7 - Manômetros e termômetros instalados a montante e a jusante do aquecedor

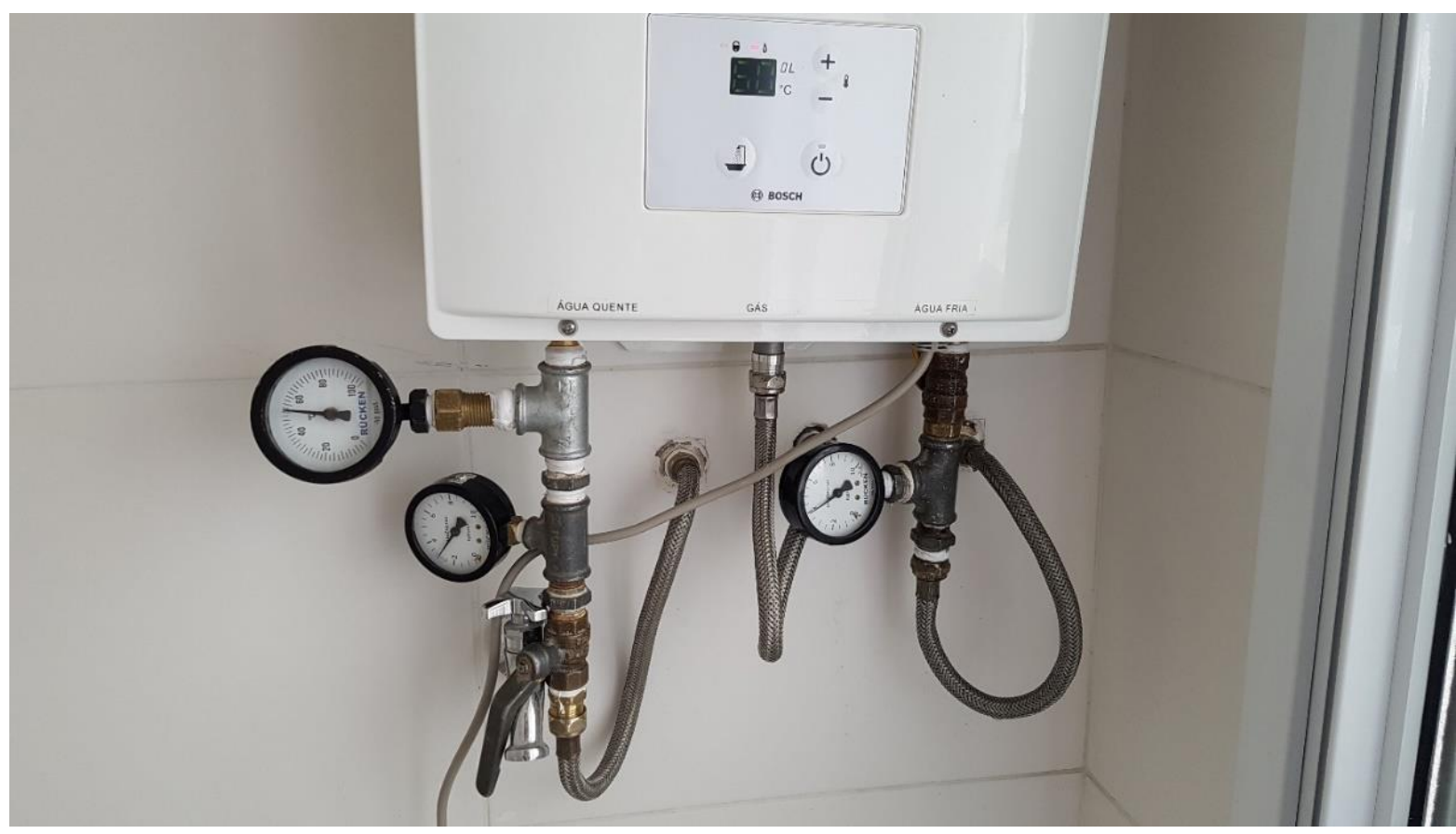

Fonte: Yamada (2021).

Os critérios de desempenho adotados estão apresentados no Quadro 2.

Quadro 2 - Critérios de desempenho adotados

\begin{tabular}{|l|l|}
\hline Critério de desempenho & Valor \\
\hline Pressão dinâmica mínima no ponto de consumo & $100 \mathrm{kPa}$ \\
\hline Faixa de vazão de cada um dos pontos a serem verificados & $0,2 \pm 0,03 \mathrm{~L} / \mathrm{s}(\mathrm{Q} n \pm 15 \%)$ \\
\hline Condição de simultaneidade prevista & dois chuveiros em uso simultâneo \\
\hline Temperatura da água quente & $43^{\circ} \mathrm{C}$ \\
\hline Temperatura da água misturada & $40^{\circ} \mathrm{C}$ \\
\hline
\end{tabular}

Fonte: Adaptador de Yamada (2021).

\section{RESULTADOS E DISCUSSÕES}

\subsection{Resultados}

Os resultados estão dispostos no Quadro 3. 
Quadro 3 - Resultados com temperatura de água quente a $43^{\circ} \mathrm{C}$ e aquecedor de $23 \mathrm{~L} / \mathrm{min}$

\begin{tabular}{|c|c|c|c|c|c|c|c|c|c|}
\hline \multirow{2}{*}{ PAV. } & \multirow{2}{*}{ AQUEC. } & \multicolumn{2}{|c|}{ PONTO DESF. } & \multicolumn{2}{|c|}{ PONTO FAV. } & \multicolumn{3}{|c|}{ RAMAL DE ALIMENTAÇÃO DAS UNI. } & \multirow{2}{*}{$\begin{array}{c}\text { ATENDE? } \\
\text { (S/N) }\end{array}$} \\
\hline & & $\underset{(L / s)}{Q}$ & $\begin{array}{c}\mathrm{Pd} \\
(\mathrm{kPa})\end{array}$ & $\begin{array}{c}Q \\
(L / s)\end{array}$ & $\begin{array}{c}\mathrm{Pd} \\
(\mathrm{kPa})\end{array}$ & $\begin{array}{l}\text { REDUTORA } \\
\quad(\mathrm{kPa})\end{array}$ & $\begin{array}{l}\text { ENTRADA AQ } \\
\quad(\mathrm{kPa})\end{array}$ & $\begin{array}{c}\text { SAÍDA AQ } \\
\text { (kPa) }\end{array}$ & \\
\hline 01 (RR) & $\begin{array}{c}\text { AQ } 23 \mathrm{~L} / \mathrm{min} \\
\left(43^{\circ} \mathrm{C}\right)\end{array}$ & 0,1551 & 75 & 0,1643 & 75 & 290 & 250 & 110 & $\mathrm{~N}$ \\
\hline $05(\mathrm{R})$ & $\begin{array}{c}\mathrm{AQ} 23 \mathrm{~L} / \mathrm{min} \\
\left(43^{\circ} \mathrm{C}\right)\end{array}$ & 0,1334 & 50 & 0,1289 & 40 & - & 150 & 70 & $\mathrm{~N}$ \\
\hline $07(\mathrm{R})$ & $\begin{array}{c}\mathrm{AQ} 26 \mathrm{~L} / \mathrm{min} \\
\left(43^{\circ} \mathrm{C}\right)\end{array}$ & 0,1174 & 40 & 0,1095 & 40 & - & 110 & 60 & $\mathrm{~N}$ \\
\hline $08(R)$ & $\begin{array}{c}\text { AQ } 23 \mathrm{~L} / \mathrm{min} \\
\left(43^{\circ} \mathrm{C}\right)\end{array}$ & 0,1172 & 40 & 0,0908 & 40 & - & 110 & 50 & $\mathrm{~N}$ \\
\hline $14(G)$ & $\begin{array}{c}\mathrm{AQ} 23 \mathrm{~L} / \mathrm{min} \\
\left(42^{\circ} \mathrm{C}\right)\end{array}$ & 0,1559 & 75 & 0,1357 & 60 & - & 350 & 140 & $\mathrm{~N}$ \\
\hline $25(P)$ & $\begin{array}{c}\mathrm{AQ} 23 \mathrm{~L} / \mathrm{min} \\
\left(43^{\circ} \mathrm{C}\right)\end{array}$ & 0,1387 & 40 & 0,1538 & 50 & - & 190 & 125 & $\mathrm{~N}$ \\
\hline
\end{tabular}

Fonte: Yamada (2021).

Nota-se, no Quadro 2, que as unidades não atendem aos critérios estabelecidos em condição de simultaneidade, mesmo com pressões elevadas na entrada. Os resultados apontam que existe uma queda acentuada na pressão dinâmica a jusante do aquecedor, seguida por uma queda da pressão dinâmica a jusante do misturador.

Considerando que a troca do aquecedor por um modelo de maior potência poderia ser uma solução, foram feitos testes com aquecedores de maior potência em algumas unidades. Os resultados estão apresentados no Quadro 4.

Quadro 4 - Resultados com temperatura de água quente a $43^{\circ} \mathrm{C}$ e aquecedor de $36 \mathrm{~L} / \mathrm{min}$

\begin{tabular}{|c|c|c|c|c|c|c|c|c|c|}
\hline \multirow{2}{*}{ PAV. } & \multirow{2}{*}{ AQUEC. } & \multicolumn{2}{|c|}{ PONTO DESF. } & \multicolumn{2}{|c|}{ PONTO FAV. } & \multicolumn{3}{|c|}{ RAMAL DE ALIMENTAÇÃO DAS UNI. } & \multirow{2}{*}{$\begin{array}{c}\text { ATENDE? } \\
\text { (S/N) }\end{array}$} \\
\hline & & $\begin{array}{c}Q \\
(L / s)\end{array}$ & $\begin{array}{c}\text { Pd } \\
(\mathrm{kPa})\end{array}$ & $\begin{array}{c}Q \\
(L / s)\end{array}$ & $\begin{array}{c}\mathrm{Pd} \\
(\mathrm{kPa})\end{array}$ & $\begin{array}{l}\text { REDUTORA } \\
\text { (kPa) }\end{array}$ & $\begin{array}{c}\text { ENTRADA AQ } \\
(\mathrm{kPa})\end{array}$ & $\begin{array}{c}\text { SAÍDA AQ } \\
\text { (kPa) }\end{array}$ & \\
\hline 02 (RR) & $\begin{array}{c}\mathrm{AQ} 36 \mathrm{~L} / \mathrm{min} \\
\left(43^{\circ} \mathrm{C}\right)\end{array}$ & 0,1603 & 60 & 0,1732 & 75 & 250 & 250 & 140 & $\mathrm{~N}$ \\
\hline $18(\mathrm{PR})$ & $\begin{array}{c}\mathrm{AQ} 36 \mathrm{~L} / \mathrm{min} \\
\left(43^{\circ} \mathrm{C}\right)\end{array}$ & 0,2157 & 170 & 0,2042 & 160 & 330 & 320 & 240 & $S$ \\
\hline
\end{tabular}

Fonte: Yamada (2021).

Observa-se que os resultados obtidos ainda não atenderam aos critérios estabelecidos.

Outra solução proposta foi aumentar a temperatura da água quente, de modo a reduzir a perda de carga do aquecedor. O Quadro 4 possui os resultados dos ensaios com uma temperatura de $60^{\circ} \mathrm{C}$. 
Quadro 5 - Resultados com temperatura de água quente a $60^{\circ} \mathrm{C}$

\begin{tabular}{|c|c|c|c|c|c|c|c|c|c|}
\hline \multirow[b]{2}{*}{ PAV. } & \multirow[b]{2}{*}{ AQUEC. } & \multicolumn{2}{|c|}{ PONTO DESF. } & \multicolumn{2}{|c|}{ PONTO FAV. } & \multicolumn{3}{|c|}{ RAMAL DE ALIMENTAÇÃO DAS UNI. } & \multirow{2}{*}{$\begin{array}{c}\text { ATENDE? } \\
\text { (S/N) }\end{array}$} \\
\hline & & $\underset{(L / s)}{Q}$ & $\begin{array}{c}\mathrm{Pd} \\
(\mathrm{kPa})\end{array}$ & $\begin{array}{c}Q \\
(L / s)\end{array}$ & $\begin{array}{c}\mathrm{Pd} \\
(\mathrm{kPa})\end{array}$ & $\begin{array}{l}\text { REDUTORA } \\
\quad(\mathrm{kPa})\end{array}$ & $\begin{array}{l}\text { ENTRADA AQ } \\
\text { (kPa) }\end{array}$ & $\begin{array}{c}\text { SAÍDA AQ } \\
\text { (kPa) }\end{array}$ & \\
\hline $05(\mathrm{R})$ & $\begin{array}{c}A Q 23 \mathrm{~L} / \mathrm{min} \\
\left(60^{\circ} \mathrm{C}\right)\end{array}$ & 0,1965 & 160 & 0,1805 & 140 & - & 240 & 200 & $\mathrm{~s}$ \\
\hline $07(\mathrm{R})$ & $\begin{array}{c}\text { AQ } 26 \mathrm{~L} / \mathrm{min} \\
\left(60^{\circ} \mathrm{C}\right)\end{array}$ & 0,1822 & 110 & 0,1796 & 110 & - & 150 & 120 & $\mathrm{~s}$ \\
\hline $14(\mathrm{G})$ & $\begin{array}{c}A Q 23 \mathrm{~L} / \mathrm{min} \\
\left(60^{\circ} \mathrm{C}\right)\end{array}$ & 0,2156 & 280 & 0,1948 & 270 & - & 400 & 300 & $S$ \\
\hline $25(P)$ & $\begin{array}{c}A Q 23 \mathrm{~L} / \mathrm{min} \\
\left(60^{\circ} \mathrm{C}\right)\end{array}$ & 0,1896 & 120 & 0,2112 & 130 & - & 190 & 140 & $\mathrm{~s}$ \\
\hline
\end{tabular}

Fonte: Yamada (2021).

Com os resultados, verifica-se que o aumento da temperatura fez com que o critério fosse atendido mesmo em pressões mais baixas.

\subsection{Discussões}

Nota-se que a exigência de vazões máximas de $0,2 \mathrm{~L} / \mathrm{s}(12 \mathrm{~L} / \mathrm{min})$ no ponto de consumo teve diversas consequências, uma vez que para o uso de limitadores de vazão, a pressão mínima no ponto para obtenção da vazão especificada é de $100 \mathrm{kPa}$. Na Figura 9 estão apresentadas as curvas de vazão de um mesmo chuveiro com e sem restritores de vazão instalados (a) e (b), respectivamente.

\section{Figura 9 - Curvas de vazão com e sem restritor de vazão}

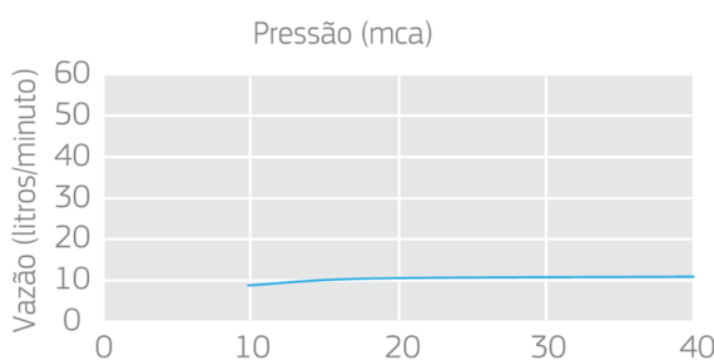

(a)

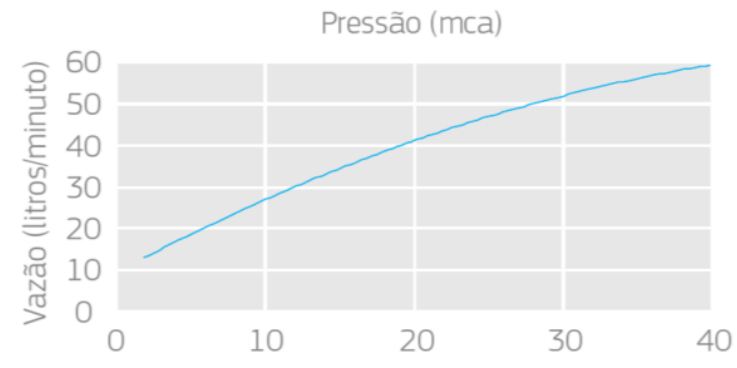

(b)

Fonte: Deca (2019)

Uma das soluções para aumentar a vazão nos pontos seria a remoção dos restritores de vazão, o que aumentaria a vazão em pressões dinâmicas mais baixas, porém essa medida pode incorrer em faixas de vazões mais amplas e em vazões que superam o valor máximo estabelecido.

Em todos os casos, observa-se que o uso de valores absolutos para pressão, vazão e temperatura não é uma prática ideal, uma vez que ao medir essas grandezas em campo existem erros causados por leitura, precisão da instrumentação empregada e diferenças nas vazões, mesmo em equipamentos sanitários iguais. 
Logo, o projeto deveria trabalhar com intervalos de pressão, vazão e temperatura ou valores nominais e tolerâncias, conforme adotado nesta pesquisa.

\section{CONCLUSÕES}

Após a execução das medições e verificações, notou-se que o método proposto permitiu a verificação das grandezas que foram definidas no critério de desempenho. As grandezas adicionais listadas, a exemplo do tempo de espera e oscilações, ainda necessitam ser estudadas e testadas, uma vez que a instrumentação em campo não permitia sua verificação.

\section{REFERÊNCIAS}

ASSOCIAÇÃO BRASILEIRA DE NORMAS TÉCNICAS. NBR 5626: Instalações prediais de água fria. Rio de Janeiro, 1998.

ASSOCIAÇÃO BRASILEIRA DE NORMAS TÉCNICAS. NBR 5626: Sistemas prediais de água fria e água quente - Projeto, execução, operação e manutenção. Rio de Janeiro, 2020.

ASSOCIAÇÃO BRASILEIRA DE NORMAS TÉCNICAS. NBR 7198: Projeto e execução de instalações prediais de água quente. Rio de Janeiro, 1993.

ASSOCIAÇÃO BRASILEIRA DE NORMAS TÉCNICAS. NBR 8130: Aquecedor de água a gás tipo instantâneo - Requisitos e métodos de ensaio. Rio de Janeiro, 2004.

ASSOCIAÇÃO BRASILEIRA DE NORMAS TÉCNICAS. NBR 15206: Instalações hidráulicas prediais Chuveiros ou duchas - Requisitos e métodos de ensaio. Rio de Janeiro, 2005.

ASSOCIAÇÃO BRASILEIRA DE NORMAS TÉCNICAS. NBR 15267: Misturadores monocomando para lavatório - Requisitos e métodos de ensaio. Rio de Janeiro, 2017.

ASSOCIAÇÃO BRASILEIRA DE NORMAS TÉCNICAS. NBR 16749: Aparelhos sanitários - Misturadores Requisitos e métodos de ensaio. Rio de Janeiro, 2019.

DECA. Catálogo Metais 2019. Acesso online. Disponível em: https://files.deca.com.br/staticfiles/catalogo/pt/deca_metais_2019_v2.pdf. Acesso em: 7 jun. 2019

YAMADA, Marco Antonio Furtado; OLIVEIRA, Lúcia Helena de. Comissionamento de sistemas prediais de água quente - Requisitos de projeto do proprietário. In: ENCONTRO NACIONAL DE TECNOLOGIA DO AMBIENTE CONSTRUÍDO, 18., 2020, Porto Alegre. Anais... Porto Alegre: ANTAC, 2020.

YAMADA, M. A. F. Comissionamento de sistemas prediais de água quente. Dissertação (Mestrado). Escola Politécnica. Universidade de São Paulo, São Paulo, 2021. 